\title{
Primary pericardial malignant mesothelioma: A case report and literature review
}

\author{
qiansu Chen ${ }^{1}$, Nailin Gong² , and Lude Liu $^{2}$ \\ ${ }^{1}$ The Affiliated Hospital of Guizhou Medical University \\ ${ }^{2}$ Guizhou Medical University
}

February 27, 2022

\begin{abstract}
Background : Primary pericardial malignant mesothelioma is a highly malignant and rare tumor. We present a case of primary pericardial malignant mesothelioma in an adult male patient and review the relevant literature to extend the understanding of the disease. Methods: The patient underwent pericardial decortication through the cardiopulmonary bypass, which effectively relieved the clinical symptoms caused by the tumor blockage. Results:Postoperative pathological diagnosis and immunohistochemistry resulted in primary pericardial malignant mesothelioma. The patient recovered after surgery and was followed up regularly after discharge. Conclusions: Primary pericardial malignant mesothelioma lacks characteristic clinical and imaging manifestations, invariably resulting in misdiagnosis. Pathological results combined with immunohistochemistry are the gold standard for diagnosis. Surgery is the mainstay of treatment, but the prognosis is extremely poor.
\end{abstract}

\section{Primary pericardial malignant mesothelioma: A case report and literature review}

Nailin Gong M.M ${ }^{1}$,Lude Liu M.M ${ }^{1}$,and Qiansu Chen M.D ${ }^{2}$

1.School of Clinical Medicine, Guizhou Medical University, Guiyang, China.

2.Department of Cardiac Surgery, Affiliated Hospital of Guizhou Medical University, Guiyang 550000, No. 28 Guiyi Street, Yunyan District, Guiyang City, Guizhou Province, China

Corresponding author: Qiansu Chen, Email:gnl709228924@126.com,Telephone numbers:+8615682327721

\section{Abstract}

Background : Primary pericardial malignant mesothelioma is a highly malignant and rare tumor. We present a case of primary pericardial malignant mesothelioma in an adult male patient and review the relevant literature to extend the understanding of the disease.

Methods: The patient underwent pericardial decortication through the cardiopulmonary bypass, which effectively relieved the clinical symptoms caused by the tumor blockage.

Results: Postoperative pathological diagnosis and immunohistochemistry resulted in primary pericardial malignant mesothelioma. The patient recovered after surgery and was followed up regularly after discharge.

Conclusions: Primary pericardial malignant mesothelioma lacks characteristic clinical and imaging manifestations, invariably resulting in misdiagnosis. Pathological results combined with immunohistochemistry are the gold standard for diagnosis. Surgery is the mainstay of treatment, but the prognosis is extremely poor.

Keywords : Primary pericardial malignant mesothelioma; cardiac surgery; pathological diagnosis; immunohistochemistry; case report 


\section{Introduction}

Primary malignant pericardial mesothelioma (PPM) is a malignant heart tumor originating in the serous pericardium and is highly invasive ${ }^{1,2}$. Through the collection of clinical data from a patient with PPM and literature review, the clinical manifestations, diagnostic methods, treatment and prognosis, pathological results and immunohistochemical characteristics of PPM are further discussed to expand the understanding of the disease and improve its diagnosis and treatment.

\section{Case report}

A 30-year-old man was hospitalized for dyspnea 1 year ago and initially diagnosed with tuberculous pericarditis. The patient regularly underwent anti-tuberculosis and other related treatments for 6 months and was discharged after symptoms were relieved. Over 50 days, the patient developed cough, sputum production, pronounced dyspnea without obvious causes, and concomitant alcoholic cirrhosis. The patient underwent enhanced computed tomography (CT), which revealed diffuse thickening of the pericardium and nodular changes (Fig. 1). Cardiovascular magnetic resonance (CMR; Fig. 2a,b) confirmed that the pericardium was diffusely thickened and abnormally strengthened, limiting cardiac contraction and extending.the mass along the left ventricular wall with possible invasion of the left heart, and tumor-like lesions were not excluded. Positron emission computed tomography suggested uneven thickening of the pericardium and increased metabolism, with infectious lesions being considered first. The patient received anti-tuberculosis therapy while reducing the burden on the heart and nourishing the myocardium by diuresis. On day 22 of the preoperative preparation, the patient suddenly suffered respiratory failure and was placed on a ventilator for endotracheal intubation. After heart failure and oxygenation indicators improved, pericardial decortication was performed. The patient was placed in a flat recumbent position and monitored intraesophageally by esophageal ultrasound. An anterior median incision of the sternum was made to enter the chest cavity, and examination revealed that the pericardium was thickened and extensively adhered, and the heartbeat was weak.Opening the pericardium longitudinally from the anterior wall of the right ventricle, the pericardium is markedly thickened (about $1.0 \mathrm{~cm}$ at the thickest point) and contractures without significant calcification. The visceral pericardium was closely adhered, and the thickened pericardium was carefully separated by gradually peeling up to the root of the aortic, down to the diaphragm, to the left side to the anterior diaphragm nerve, and to the right side to the atrium in the order of the outflowing tract first and then into the tract. The thickened visceral pericardial tissue at the apex of the heart was tightly adhered to the heart and was released where it could not be peeled off. The area of excision and release of the pericardium was approximately $15 \mathrm{~cm} \times 10 \mathrm{~cm}$. Cardiac beats were seen to be significantly strengthened, and central venous pressure was slightly lower than before surgery. The surgery was effective, and the patient was treated in intensive care after surgery and underwent three rounds of artificial liver therapy for liver failure and ventilator-assisted therapy for 8 days. The patient was not treated again for tuberculosis, and did not receive tumor-related treatment owing to liver failure and poor systemic condition. He was discharged from the hospital 44 days after surgery. Postoperative pathological results revealed pericardial fibrous tissue hyperplasia and endothelial cell hyperplasia in the shape of a cord (Fig. 3a,b). Mesothelial cell atypia was not pronounced. Hyperplastic mesothelial cell cords were arranged in some areas, and mucoid degeneration could be observed around them. In the immunohistochemical results, adenoid structural cells were CK(+), EMA $($ partial + ), CK5 $/ 6(+), \operatorname{HBME}-1(+)$, calretinin $(+)$, WT1 $(+)$, D2-40 $(+)$, CEA $(-)$, TTF-1 $(-)$, CK7 $(+)$, CK20(-), CDX-2(-), CD31(-), CD34(-), FLi1 (+), p53 (partial+), Vim (+). ), p16(-), GLUT1 (partial +), desmin(-), Ki-67 (approximately 20\% +) (Fig. 4a,b,c,d). Histological images combined with marker results resulted in a diagnosis of pericardial-reactive mesothelial cell hyperplasia and pericardial malignant mesothelioma (epithelial-like type).

\section{Discussion}

\subsection{Clinical manifestations of PPM}

The incidence of PPM is extremely low, with multiple occurrences in middle-aged men and an unknown etiology ${ }^{3}$.PPM has no characteristic clinical manifestations, and the main symptoms are dyspnea, chest 
tightness, chest pain, cough, sputum, fever, and night sweats. The main signs of PPM include enlargement of the heart, distant heart sounds, pericardial percussion, and various signs of systemic congestion. PPM is often misdiagnosed as tuberculous pericarditis and constrictive pericarditis ${ }^{4}$. There was no history of asbestos exposure in this case. The patient had no obvious abnormalities in liver function 1 year ago, and long-term alcohol consumption led to alcoholic cirrhosis. Pericardial disease led to right heart failure, which accelerated the process of liver failure.

\subsection{Imaging diagnosis of PPM}

Echocardiography is the most frequently used method of evaluation of PPM and can identify the location and morphology of a tumor and assess the effect of the tumor on cardiac hemodynamics and determine the hypocardial function ${ }^{5}$. However, PPM lacks characteristic echocardiographic imaging changes, and its diagnosis is influenced by the experience and subjectivity of the ultrasound physician. Cardiac CT is better than echocardiography for observing pericardial changes, and enhanced scans can determine the condition of the large blood vessels of the heart. Studies have shown that low-density areas suddenly appear within the thickened pericardium, which may be associated with necrosis inside the tumor ${ }^{6}$. In the current case, cardiac CT showed marked pericardial diffuse thickening with nodular changes and decreased density of the heart chambers and large blood vessels, consistent with these studies. CMR can identify the surrounding soft tissue invasion and allows a reliable judgment on the nature of the pericardial tumor, the degree of myocardial infiltration, and the location ${ }^{7}$. PET-CT provides detailed molecular information about the function and metabolism of lesions, allowing for the early detection of pericardial tumors, and can determine benign and malignant nature and metastasis ${ }^{8}$. In summary, imaging data is conservative regarding the diagnosis of PPM, and generally prioritizes pericarditis in terms of imaging performance. Even with optimal cardiac CMR and PET-CT, there are often no characteristic changes and lesions are rarely considered as pericardial tumors, which is the main cause of preoperative misdiagnosis or missed diagnosis.

\subsection{Pathological results and immunohistochemical analysis of PPM}

Postoperative pathology combined with immunohistochemical analysis is the gold standard for diagnosis of $\mathrm{PPM}^{9}$. The anatomical typing of PPM includes diffuse and focal types, with diffuse types being more common. Histological classifications are epithelial, sarcoma, and bipolar, with epithelial being the most common $^{10}$. Currently, combined immunohistochemistry showing negative expression of CKp (AE1/AE3), CK5/6, CK18/19, EMA, MC, wave protein (vimentin), calretinin, carcinoembryonic antigen (CEA), and thyroid transcription factor is recommended for $\mathrm{PPM}^{2,9-14}$. The immunohistochemical expression signature of PPM is mostly the permutation and combination of the above antibodies. This case also confirmed the results of the above pathological typing and immunohistochemical analysis, consistent with the diagnosis of PPM.

\subsection{Treatment and prognosis of PPM}

Surgery is the main treatment for primary cardiac malignancy, which not only removes tumor tissue and obtains material for the pathological diagnosis, but also relieves clinical symptoms caused by tumor blockage ${ }^{15}$. Because PPM is rare, a unified treatment specification has not been determined clinically. However, in PPM treatment, surgery is critical for solving pericardial thickening, adhesions, and restoring cardiac contractile function ${ }^{16}$. When the patient has life-threatening comorbidities, surgery can relieve pericardial compression, prolong survival time, and improve quality of life. In the current case, the surgical effect was remarkable, successfully relieving the pericardium from extensive thickening and adhesions. The patient was alive at follow-up at 3 months postoperatively. Postoperative combination of chemotherapy and radiation therapy may improve prognosis, but long-term efficacy remains poor. However, chemotherapy after cardiac malignancy resection should be beneficial ${ }^{17}$. PPM treatment is trending towards a combination of surgery, radiotherapy, chemotherapy, and other comprehensive treatment measures.

PPM has extremely low incidence, atypical clinical manifestations, difficult early diagnosis, and poor prognosis. Nevertheless, clinicians should extend their understanding of the disease and strive to achieve early diagnosis, early intervention, and improve prognosis to enhance patient survival. 


\section{ACKNOWLEDGMENTS}

We thank H. Nikki March, PhD, from Liwen Bianji (Edanz) (www.liwenbianji.cn/), for editing the English text of a draft of this manuscript.

\section{CONFLICT OF INTERESTS}

The authors declare no conflict of interest.

\section{ETHICS AND LEGAL}

IRB approval, clinical trial statement, and registration details are N/A. Informed consent was obtained from both the patient and their legal guardian for this case report.

\section{AUTHOR CONTRIBUTIONS}

Qiansu chen - Concept/Design, critical review of draft, case analysis, and interpretation.

Nailin Gong and Lude Liu have equal contributions to this article. - Case analysis and interpretation, report drafting, and revisions.

\section{REFERENCES}

1 Isoda R, Yamane H, Nezuo S, et al. Successful palliation for an aged patient with primary pericardial mesothelioma. World J Surg Oncol, 2015, 13: 273.

2 Matsuyama S, Imazuru T, Uchiyama M, Ota H, Iida M, Shimokawa T. Primary malignant pericardial mesothelioma presenting with cardiac tamponade. Int J Surg Case Rep. 2020;73:253-256.

3 Mensi C, Giacomini S, Sieno C, et al. Pericardial mesothelioma and asbestos exposure. Int J Hyg Environ Health, 2011, 214: 276-279.

4 Fujita K, Hata M, Sezai A, et al. Three-year survival after surgery for primary malignant pericardial mesothelioma: report of a case. Surg Today, 2014, 44: 948-951.

5 Adler Y, Charron P, Imazio M,et al. 2015 ESC Guidelines for the diagnosis and management of pericardial diseases: the task force for the diagnosis and management of pericardial diseases of the European Society of Cardiology (ESC). Eur Heart J 2015;36:2921-2964.

6 Peebles CR, Shambrook JS, Harden SP. Pericardial disease-anatomy and function.

Br J Radiol 2011;84 Spec No 3(Spec Iss 3):S324-S337.

7 Pennell DJ, Sechtem UP, Higgins CB, Manning WJ, Pohost GM, Rademakers FE,

van Rossum AC, Shaw LJ, Yucel EK. Clinical indications for cardiovascular magnetic resonance (CMR): consensus panel report. Eur Heart J 2004;25:1940-1965

8 Suman S, Schofield P, Large S. Primary pericardial mesothelioma presenting as pericardial constriction: a case report. Heart 2004;90:e4.

9 McGehee E, Gerber DE, Reisch J, Dowell JE. Treatment and outcomes of primary pericardial mesothelioma: a contemporary review of 103 published cases. Clin Lung Cancer. 2019;20(2): e152-e157.

10 Xing Rongge,Zhang Xin,Zhang Xiaoling,et al. Clinical pathological features of pleural malignant mesothelioma and related antibodies [J]. Hebei Medical,2010,32( 8): 934-936.

11 CEN Yulan. Clinical pathological analysis of 20 cases of malignant mesothelioma[J]. Clinical Review, 2008, 23( 1): 43-44. 
12 Kong Lingyun, Wang Jingrui, Sun Lanlan, Zhu Weiwei, et al. pathological and prognostic summary analysis of primary malignant pericardial mesothelioma in Chinese mainland 35 years[J].Chinese Journal of Circulation,2017,32(10):1005-1009.

13 Tang Qile,Xu Li,Xu Wenjuan,et al. Clinicopathological analysis of pericardial primary malignant mesothelioma[J]. Journal of Clinical and Experimental Pathology,2001,17(5): 400-403.

14 HUSAIN A N, COLBY T V, ORDONEZ N G, et al.Guidelines for pathologic diagnosis of malignant mesothe-lioma 2017 update of the consensus statement from the international mesothelioma interest group[J]. Arch Pathol Lab Med, 2018, 142(1): 89-108. [15] JOVY, CIBAS E S, PINKUS G

15 Apicella G, Boulemden A, Citarella A, et al.Surgical treatment of a primary malignant pericardial mesothelioma: case report. Acta Chir Belg. 2022 Feb;122(1):48-50.

16 Fujita K, Hata M, Sezai A, et al. Three-year survival after surgery for primary malignant pericardial mesothelioma: report of a case. Surg Today, 2014, 44: 948-951.

17 Agarwal V,Agarwal S,Srivastava AK,et al. Primary cardiac tumors: surgical experience and follow up. Indian Heart J,2003,55: 632-636.

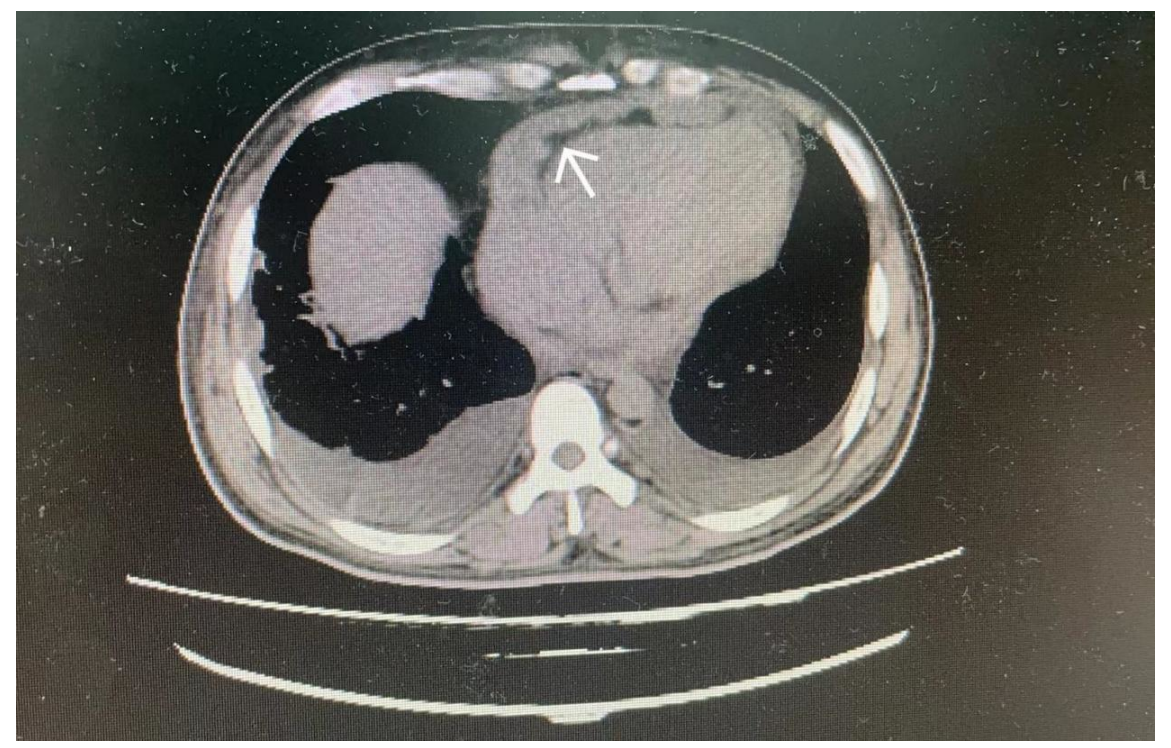

Figures

Fig. 1

CT: arrows are diffuse thickening of the pericardium and nodular changes. Bilateral pleural effusion. 

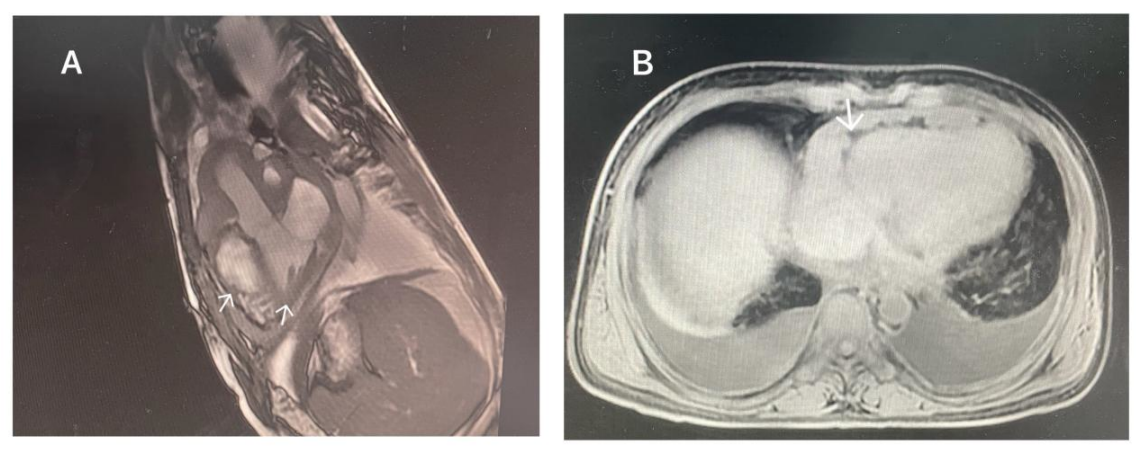

Fig. 2

CMR:The arrows are diffuse thickened pericardium, and the mass may be seen invading the left ventricle

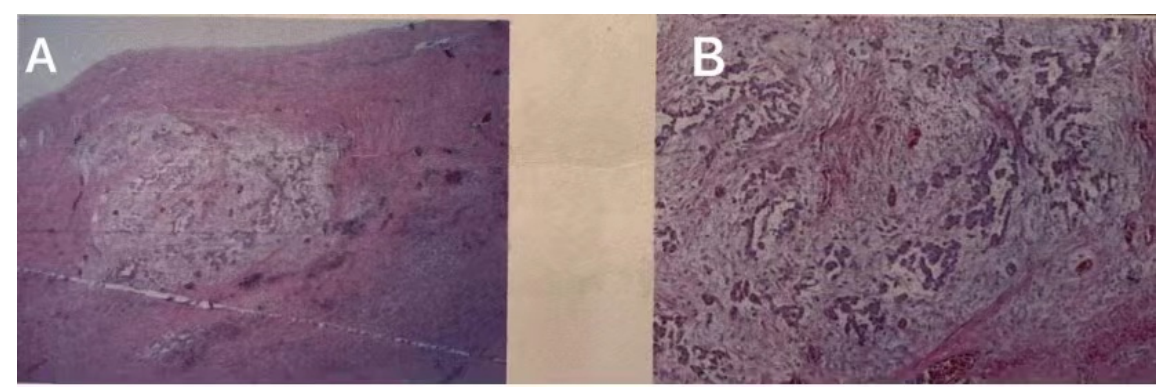

Fig. 3

HE stained images of PPM specimens
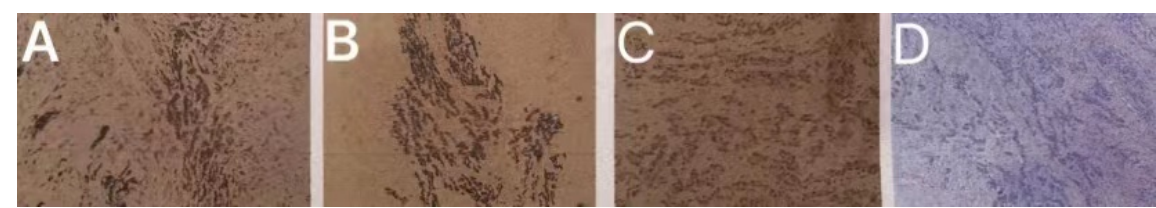

Fig. 4

Images of postoperative immunohistochemical results of PPM 